\title{
LOAD EFFECT ON THE STARTING PERFORMANCE OF A STANDING WAVE THERMOACOUSTIC ENGINE
}

\author{
C. Weisman ${ }^{1}$, D. Baltean-Carlès ${ }^{1 *}$, I. Delbende ${ }^{1}$, L. Ma $^{1}$, and L. Bauwens ${ }^{2}$ \\ ${ }^{1}$ Université Pierre et Marie Curie UPMC Sorbonne Universités, Laboratoire d'Informatique pour la Mécanique et les \\ Sciences de l'Ingénieur Orsay, France. *Corresponding author's e-mail: baltean@limsi.fr \\ ${ }^{2}$ Department of Mechanical and Manufacturing Engineering, University of Calgary, Canada.
}

Keywords: standing wave engine, resistive load, numerical simulation, low Mach number approximation, thermoacoustic instability

\section{Introduction}

A thermoacoustic engine is a device absorbing heat at the hot heat exchanger and releasing heat at the cold heat exchanger while producing acoustic work as an output. Engines are built to carry a load, which in the case of an acoustic device, might include a thermoacoustic refrigerator [4], a (possibly linear) electric generator [2] etc. Coupling between the engine and the load influences both starting performance and steady operation.

The aim of the current work is to study the influence of a variable load on the onset conditions of a standing-wave thermoacoustic engine, through direct numerical simulation of the starting phase. Unstable acoustic modes develop spontaneously in the system. The computed acoustic pressure signal in the active cell is analyzed in order to extract the growth rate and frequency of the dominant modes. Therefore the critical hot exchanger temperature and frequency of the associated mode allowing the engine to start can be determined.

\section{Physical model and numerical simulation}

The geometry consists of a long tube, within which an active cell is placed. Flow in the resonator is then described by linear acoustics. The solutions are expressed as a pair of planar traveling waves that move respectively left and right at the speed of sound, using Riemann invariants. The active cell consists of a stack of horizontal solid plates, placed between two heat exchangers also made of solid horizontal plates that have the same periodicity as the stack. There is no empty space between stack and heat exchanger plates, resulting in an optimal configuration for the engine. The geometry can be reduced to a domain consisting of two halfplates plus the gap between them, plus a region that extends away from the solid plates.

Flow in the active cell is described by the two-dimensional Navier-Stokes equations in the low Mach number approximation [1]. The solid stack plate problem is described by the heat conduction equation. In the stack and heat exchanger plates, thermophysical properties of the gas are taken to be constant. In the heat exchangers, uniform, constant temperatures are imposed with dimensionless values $T_{c}=1$ in the cooler plates, $T_{h}>1$ in the heater plates. Given the periodicity, open active cell boundaries are effectively adiabatic. The flow in the active cell is coupled to linear acoustics model in the resonator, using matched asymptotic expansions in the low Mach number limit [1]. The flow boundary conditions at resonator ends (closed end and loaded end) are then transported along the characteristics to the open end locations of the active cell.

The load is taken to be a resistive load. Instantaneous acoustic pressure and velocity are assumed to be in phase at the loaded end and the load is thus reduced to a real scalar value $f$ measuring the ratio of velocity over acoustic pressure. That condition, carried along the char- 
acteristics, results in a relation between the Riemann invariants at the right end of the active cell through a real coefficient $Z=(\gamma-f) /(\gamma+f)$, where $\gamma$ is the ratio of the gas specific heats. While $f$ goes from zero to infinity, $Z$ has the finite span from -1 for a closed end to +1 for an open end, and zero for $f=\gamma$. In the framework of the harmonic approximation, it is possible to relate $f$ or $Z$ to the reflection coefficient at the loaded end hence to the standing wave ratio [3]. The limit values $Z= \pm 1$ correspond to a pure standing wave in the resonator, while the limit case $Z=0$ corresponds to a pure traveling wave moving from the active cell towards the load. Between those limit cases, the wave is a combination between standing and traveling waves.

\section{Results}

The problem in the active cell is solved numerically using a finite volume code [1]. Numerical simulations are carried out for an existing prototype of thermoacoustic engine [1]. The acoustic pressure signal from each simulation is then analyzed. The growth rate and frequency of the dominant modes are extracted from the signal, yielding the critical temperature $T_{h}$ for which the first unstable mode appears and the frequency of that mode.

Varying the load and reproducing the same procedure for each load value results in a stability diagram, Figure 1 (left), in the $\left(Z, T_{h}\right)$ plane, showing stable and unstable zones. The region above the stability curve corresponds to instability. Results show that for a wide range of intermediate load values (for $Z$ close to zero), the critical temperature ratio is so high that in practice the engine will not start on its own. Figure 1 (right) shows the dimensionless angular
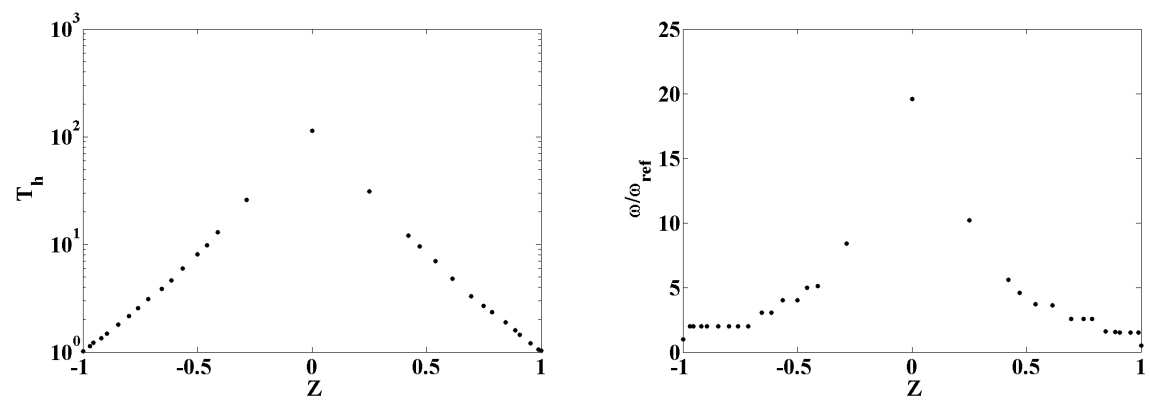

Figure 1: Left: Stability diagram in the $\left(Z, T_{h}\right)$ plane. The region above the curve corresponds to instability. Right: Angular frequency of the most unstable mode scaled by $\omega_{\text {ref }}$, as a function of $Z$.

frequency $\omega / \omega_{\text {ref }}$ of the first mode to become unstable as a function of $Z$. Critical modes near $Z=-1$ are of the closed-end type (multiples of $\lambda / 2$ ) whereas critical modes near $Z=1$ are of the open-end type (odd multiples of $\lambda / 4$ ). As $|Z|$ decreases, the frequency of the most unstable mode is observed to increase, and higher harmonics are first destabilized. This results in distinct sets of onset frequencies, each set corresponding to a specific harmonic. The load influences the wavelength and changes the optimal relative stack position, so that the most unstable mode shifts from fundamental to first harmonic, second harmonic and so on. These results were found to be in good agreement with an analysis based on Rott's linear theory.

\section{References}

[1] Hireche, O., Weisman, C., Baltean-Carlès, D., Le Quéré, P., and Bauwens, L. "Low Mach number analysis of idealized thermoacoustic engines with numerical solution". J. Acoust. Soc. Am., 128, (2010), 3438-3448.

[2] Miglori, A. and Swift, G. "Liquid sodium thermoacoustic engine". Appl. Phys. Lett., 53, (1988), 355-357.

[3] Raspet, R., Bruster, J., and Bass, H. "New Approximation Method for Thermoacoustic Calculations". J. Acoust. Soc. Am., 103, (1998), 2395-2402.

[4] Swift, G. “Thermoacoustic engines”. J. Acoust. Soc. Am., 84, (1988), 1145-1180. 